\title{
Telefon-Triage durch Krankenkassen - eine Sparmassnahme?
}

\author{
P. Ackle, K. Bamert, \\ G. Dufner, M. Enz Kuhn, \\ K. Kaspar, M. P. Mahler, \\ R. Mahler, B. Rickenbacher, \\ R. Rüegg, A. Weisshaar
}

Qualitätszirkel Argonet
Korrespondenz:

Dr. med. Kurt Kaspar Zentrum Gugger

CH-5442 Fislisbach

kurt.kaspar@hin.ch

www.argomed.ch

\section{Ausgangslage}

Anlässlich der zu erwartenden überdurchschnittlichen Prämienerhöhung werden von Bundesrat und Parlament, von santésuisse und verschiedenen anderen interessierten Kreisen Sparvorschläge präsentiert. Einige von diesen sind sicher sinnvoll, andere sind eher fragwürdig. Als Hausärzte, engagiert an der Basis tätig, glauben wir nicht, dass der Aufbau einer zusätzlichen Struktur, nämlich der von den Krankenversicherern obligatorisch anzubietenden Telefontriage, die erwarteten Einsparungen bringen wird.

Leider ist in der politischen Öffentlichkeit nur wenig über die allgemeinen Leistungen bekannt, die von den Hausarztpraxen meist unentgeltlich erbracht werden. Neben den Aktivitäten im sozialen Bereich, in Vereinen, beim Sammeln von Daten für das BAG und anderen mehr ist die Telefonberatung durch unsere gut ausgebildeten MPAs eine weitere wichtige Tätigkeit. Ziel unserer Qualitätszirkel-Arbeit war es, diesen Dienst an unseren Patienten zu dokumentieren.

\section{Vorgehen}

Alle 10 Mitglieder unseres Qualitätszirkels haben sich verpflichtet, durch ihre MPAs sämtliche Telefonate während 1-3 Wochen zu dokumentieren. Dabei wurde auf einer einfachen Strichliste jedes Telefongespräch notiert. Es sind keine nennenswerten Probleme bei der Dokumentation und der Auswertung aufgetreten. Wir haben unterschieden zwischen den Kategorien: Vergeben eines Konsultationstermins, administrative Fragen, abschliessende Beratung durch die MPA, Anforderung eines Rezeptes, Zeugnisses usw.

\section{Resultate}

1340 Telefonanrufe konnten ausgewertet werden. 15,7 Prozent der Anrufer erhielten einen Termin am gleichen Tag. 16 Prozent der Anrufer wurden medizinisch abschliessend von der MPA beraten, $5 \%$ davon nach Rücksprache mit dem Arzt. In 3,7\% war dadurch eine Konsultation nicht mehr notwendig.

\section{Diskussion}

Das Resultat zeigt, dass unsere MPAs häufig die Anrufenden abschliessend beraten. Dies ist möglich, weil sie viele der Hausarztpatienten kennen, was eine persönliche telefonische Betreuung ermöglicht. Wünsche nach einem Rezept sind einfach erfüllbar, weil die Krankenakten und damit die Anamnese der Patienten vorliegen. Auch kurze Rückfragen beim Arzt sind möglich, was in 5\% der Beratungen notwendig war.
Gemäss unserer Erhebung in recht unterschiedlichen Arztpraxen mit verschiedenen Schwerpunkten erhalten 15,7\% der Patienten am gleichen Tag einen Termin. Dies ist selbstverständlich nur möglich, wenn die MPA eine gute Triage macht und die Dringlichkeit richtig einschätzt. Regelmässige Fortbildungen in den Praxen und in Berufsverbänden haben zu einem sehr guten medizinischen Wissensstand der medizinischen Praxisassistentinnen geführt. Die erwähnten Dienstleistungen werden aus dem allgemeinen Praxiseinkommen finanziert. Eine Tarifposition für die Beratung durch die MPA existiert in TARMED nicht.

Telefonberatungen werden von einigen Krankenkassen schon länger angeboten. In der Öffentlichkeit ist nichts über die Kosten dieser Dienstleistung bekannt. Es ist nicht publiziert, wie viel die Krankenversicherer den Beratungs- und Triageunternehmungen bezahlen. In unseren Allgemeinpraxen fallen vorwiegend die Fälle auf, in denen die Patienten dann doch zum Arzt kommen, und zwar nicht selten unnötigerweise. Die abschliessend Beratenen sehen wir nicht.

\section{Schlussfolgerungen}

Einmal mehr zeigt sich eine Diskrepanz zwischen dem Lippenbekenntnis, die Hausärzte zu fördern und den Taten im politischen Alltag. Während Laborpositionen unter ihren Gestehungswert gekürzt werden mit dem Hinweis, es dürfe keine Quersubvention der Praxis durch die Laboranalytik stattfinden, ist im vorliegenden Fall eine Subvention der Dienstleistung aus der Tasche des Arztes offenbar erwünscht. «Neue Methoden» wie Telefontriage, Telemedizin werden schnell in die Tarife integriert, wohl, weil sie spektakulärer

\begin{tabular}{|c|c|c|}
\hline Telefongrund & Zahl gesamt & Prozent \\
\hline Normale Konsultation & 394 & 29,4 \\
\hline Konsultation gleichentags & 210 & 15,7 \\
\hline Telefonische Konsultation & 79 & 5,9 \\
\hline Administration & 327 & 24,4 \\
\hline $\begin{array}{l}\text { Medizin. Beratung durch } \\
\text { MPA }\end{array}$ & 100 & 7,4 \\
\hline $\begin{array}{l}\text { Beratung nach Rücksprache } \\
\text { mit Arzt }\end{array}$ & 67 & 5,0 \\
\hline $\begin{array}{l}\text { Beratung verhindert } \\
\text { Konsultation }\end{array}$ & 50 & 3,7 \\
\hline Rezept & 114 & 8,5 \\
\hline Total & 1340 & 100 \\
\hline
\end{tabular}


sind als die altbekannte Hausarzttätigkeit. Diese gerät damit in der Wertschätzung zunehmend in den Hintergrund.

Wenn mit Blick auf Kosteneinsparungen die Telefontriage obligatorisch wird, verlangen wir die Offenlegung der Kosten derselben. Wie in der Schweiz üblich, wird jeder Krankenversicherer einen eigenen Apparat aufziehen und den wohl auch zumindest kostendeckend betreiben wollen. Umso wichtiger ist das Kosten-Monitoring, denn Telefontriage ist anspruchsvoll, speziell bei nicht bekannten Patienten. Es braucht dazu einen personellen und technischen Aufwand, den bisher in der Schweiz nur einige wenige Unternehmen mit guter Qualität anbieten können. In unseren Praxen wurden 16\% der Anrufenden abschliessend beraten, wohl meist ohne Kostenfolge. Die Gegenüberstellung mit dem neuen Triage-Apparat erwarten wir mit Spannung.
Eine Möglichkeit, einen Einspareffekt mit bestehenden Strukturen zu erzielen, wäre, die Hausärzte und ihr Praxispersonal, die in Triage jahrelang geschult sind, für diese Funktion einzusetzen. Das hiesse aber, sie dafür auch zu entschädigen. Für Krankenversicherer ist es möglich, Pauschalabgeltungen und Entschädigungen pro Telefonanruf an die Beratungsunternehmen zu bezahlen. Wieso sollte das nicht für die Hausarztpraxis möglich sein? Wieso kann der Hausarzt nicht unbürokratisch für diese Leistung entschädigt werden, damit er die dazu notwendige Zeit und das Personal auch bereitstellen kann? Über den üblichen TARMED-Tarif soll diese Leistung nicht abgerechnet werden, da viel zu viele kleine Rechnungen mit entsprechendem Aufwand generiert würden. Ziel der Telefontriage müsste es sein, unnötige Konsultationen mit entsprechenden Kostenfolgen zu verhindern. Unser Praxispersonal macht dies seit Jahren.

\section{Sie lesen gerade eine Zeitschrift des Schweizerischen Ärzteverlags EMH ...}

\section{Wussten Sie schon,}

- dass EMH ein Gemeinschaftsunternehmen der Verbindung der Schweizer Ärztinnen und Ärzte FMH und der Schwabe AG ist, dem mit Gründung 1488 ältesten Druck- und Verlagshaus der Welt?

- dass EMH mit insgesamt zehn Fachzeitschriften, einem umfangreichen Online-Angebot sowie einem wachsenden Buchprogramm der führende Verlag für medizinische Zeitschriften in der Schweiz ist?

- dass sämtliche bei EMH erscheinenden Zeitschriften offizielle Publikationsorgane der jeweils zuständigen medizinischen Fachorganisationen sind?

Bei EMH werden Publikationen von Ärztinnen und Ärzten für Ärztinnen und Ärzte herausgegeben. Die unabhängigen Redaktionen mit namhaften Fachpersonen aus der medizinischen Praxis gewährleisten die hohe Qualität und Relevanz der Beiträge, die das gesamte Spektrum der medizinischen Diskussion abdecken. Die Themenwahl orientiert sich nicht daran, wo Werbegelder der Industrie vorliegen, sondern spiegelt die vielfältigen Interessen der Ärzteschaft sowie der Fachorganisationen wider.

Wenn Sie mehr über EMH wissen möchten, finden Sie unter www.emh.ch weitere Informationen sowie die Online-Ausgaben unserer Zeitschriften mit komfortabler Volltext-Suche.

Gerne stehen wir Ihnen für Ihre Fragen auch persönlich zur Verfügung:

EMH Schweizerischer Ärzteverlag AG Farnsburgerstrasse 8

$\mathrm{CH}-4132$ Muttenz
Marketing und Kommunikation

Tel. +41 (0)61 4678506 / Fax +41 (0)61 4678556

marketing@emh.ch

\section{EMH Schweizerischer Ärzteverlag - Publikationen am Puls der Medizin}

